\title{
Identification of amino acid residues required for ferric-anguibactin transport in the outer-membrane receptor FatA of Vibrio anguillarum
}

Correspondence

Jorge H. Crosa

crosajor@ohsu.edu

Received 24 August 2006

Revised 24 October 2006

Accepted 2 November 2006
Claudia S. López, ${ }^{1}$ Alejandro F. Alice, ${ }^{1}$ Ranjan Chakraborty ${ }^{2}$ and Jorge $\mathrm{H}$. Crosa ${ }^{1}$

\author{
${ }^{1}$ Department of Molecular Microbiology and Immunology, Oregon Health and Science \\ University, Portland, OR 97239, USA \\ ${ }^{2}$ Department of Health Sciences, College of Public and Allied Health, East Tennessee State \\ University, Johnson City, TN, USA
}

\begin{abstract}
Vibrio anguillarum 775 is a fish pathogen that causes a disease characterized by a fatal haemorrhagic septicaemia. It harbours the $65 \mathrm{kbp}$ pJM1 plasmid, which encodes an iron sequestering system specific for the siderophore anguibactin and is essential for virulence. The genes involved in the biosynthesis of anguibactin are located on both the pJM1 plasmid and the chromosome. However, the genes for the outer-membrane receptor FatA and the other transport proteins are only carried on the plasmid. With the aim of elucidating the mechanism of ferricanguibactin transport mediated by FatA, this work focuses on the identification of Fat $A$ amino acid residues that play a role in the transport of ferric-anguibactin, by analysing the transport kinetics of site-directed mutants. The mutations studied were located in conserved residues of the lock region, which contains a cluster of ten residues belonging to the $\mathrm{N}$-terminal and barrel domains, and of the channel region of FatA, which contains conserved glycines located in the $\beta 5-\beta 6$ loop and a conserved arginine located in strand 11 of the $\beta$-barrel. In the case of the FatA lock region, it is clear that although the residues analysed in this work (R95, K130, E505 and E550) are conserved among various outer-membrane receptors, their involvement in the transport process might differ among receptors. Furthermore, it was determined that in the FatA channel region double substitutions of the conserved glycines 131 and 143 with alanine resulted in a variant receptor unable to transport ferric-anguibactin. It was also shown that the conserved arginine 428 located in strand 11 is essential for transport. The results suggest that a conformational change or partial unfolding of the plug domain occurs during ferric-anguibactin transport.
\end{abstract}

\section{INTRODUCTION}

In the host and in natural environments, ferric iron is unavailable since under aerobiosis at biological $\mathrm{pH}$ it is quite insoluble (free iron $10^{-18} \mathrm{M}$ ). In the vertebrate host the majority of this iron is found in red blood cells or bound by host proteins, such as transferrin and lactoferrin (Bullen \& Griffiths, 1999). Therefore, during infection bacterial pathogens depend on their ability to scavenge this essential metal from the host-complexed state. These types of invasive organisms have the ability to produce low-molecular-mass iron sequestering molecules with high affinity for ferric iron, known as siderophores. These compounds are synthesized from carboxylic acids and amino acids via a nonribosomal peptide synthetase mechanism and contain side chains and

Abbreviations: EDDHA, ethylenediamine-di-(o-hydroxyphenylacetic) acid; ITB, iron transport biosynthesis; $K_{\mathrm{D}}$, dissociation constant; $K_{\mathrm{m}}$, Michaelis constant; SOE, splicing by overlap extension. functional groups able to coordinate ferric ions with high affinity and specificity (Crosa \& Walsh, 2002).

Vibrio anguillarum is a Gram-negative, polarly flagellated, comma-shaped rod bacterium that is responsible for both marine and freshwater fish epizootics all over the world. This bacterium causes a highly fatal haemorrhagic septicaemic disease not only in salmonids, but also in other types of fish, including eels (Actis et al., 1999). Many virulent serotype $\mathrm{O} 1$ strains harbour the $65 \mathrm{kbp}$ pJM1 virulence plasmid, which encodes an iron-sequestering system essential for their pathogenicity (Crosa, 1980). This system includes most of the biosynthetic genes for the siderophore anguibactin, as well as the transport proteins FatA, B, C and D, involved in the transport of ferricanguibactin into the cytoplasm (Actis et al., 1988; Crosa, 1980; Koster et al., 1991; Tolmasky et al., 1988). This peptide siderophore is composed of one molecule each of 2,3dihydroxybenzoic acid (DHBA), L-cysteine and N-hydroxyhistamine (Actis et al., 1986; Jalal et al., 1989). 
The ferric-siderophore's receptors are specific in the recognition of these iron complexes, with the energydependent transport being driven by the proton-motive force across the cytoplasmic membrane (Bradbeer, 1993; Kadner, 1990). The energy transduction from the cytoplasmic membrane to the outer membrane requires the TonB, ExbB and ExbD proteins (Bassford et al., 1976; Fischer et al., 1989; Frost \& Rosenberg, 1975; Wiener, 2005). The TonB protein, which is anchored in the cytoplasmic membrane, extends into the periplasm and interacts with the receptor during transport of the ferric-siderophore complex, transducing the energy from the cytoplasmic membrane; however, the exact mechanism of this energy transduction event is still unknown (Postle \& Kadner, 2003). In the case of $V$. anguillarum two TonB systems are present but only one, the TonB2 system, operates in ferric-anguibactin transport (Stork et al., 2004).

The structures of several outer-membrane receptors have been solved, including those of FecA, FepA, FhuA and BtuB from Escherichia coli and FpvA and FptA from Pseudomonas aeruginosa (Buchanan et al., 1999; Chimento et al., 2003; Cobessi et al., 2005a, b; Ferguson et al., 1998, 2002). More recently, the crystal structure of the E. coli TonB protein in complex with the cobalamine receptor BtuB (Shultis et al., 2006) and the ferrichrome receptor FhuA (Pawelek et al., 2006) have also been solved. Taking into account these structures, several laboratories have identified specific amino acids intervening in the binding and/or transport of ferric-siderophore complexes (Cao et al., 2000; Chakraborty et al., 2003; Endriss et al., 2003; Mislin et al., 2006; Sauter \& Braun, 2004; Shen et al., 2005). From these structures it was possible to determine that on the extracellular side, the $\beta$ strands of the barrel are connected to form solvent-accessible extracellular loops of variable lengths, while on the periplasmic side relatively shorter loops are present (van der Helm, 2004). The extracellular loops form a pocket to receive the ferric-siderophore complex from the external environment and there are no homologies observed in these loops or the apices formed by the N-terminal domain, which is consistent with their recognizing and binding to chemically and structurally diverse siderophores. Moreover, by sequence alignment of several receptors, it can be deduced that most of the conserved residues are in the $\mathrm{N}$-terminal region, forming a plug domain, with the remainder located in the portion of the barrel embedded in the membrane (Chimento et al., 2005; Ferguson \& Deisenhofer, 2002; van der Helm, 2004). The plug, formed by approximately $150 \mathrm{~N}$-terminal residues, consists of a four-stranded mixed $\beta$ sheet inclined by about $45^{\circ}$ with respect to the membrane plane (Chakraborty et al., 2003; Ferguson \& Deisenhofer, 2002; Ferguson et al., 2002). The ferric-siderophore binding site involves amino acid residues located in the external loops as well as some residues from the three apices of the $\mathrm{N}$-terminal plug domain. In FecA, the diferric-dicitrate receptor, the interaction of the ligand complex with the receptor significantly affects the conformation of the external loops as well as the plug domain (Ferguson et al., 2002; Yue et al., 2003). The major structural changes observed upon binding of the ferric-siderophore are in loops 7 and 8 and minor changes were also observed in loops 4, 5 and 9 (Ferguson et al., 2002). However, this conformational change has not been observed in the other ligand-bound structures. Binding of the ligand to FecA also induces conformational changes in the plug domain: apices $\mathrm{A}$ and $\mathrm{C}$ shift toward the diferricdicitrate molecule, apex B shifts away from the siderophore, and there is a concerted downward movement of $\beta 5$ and $\beta 6$ toward the periplasm (Ferguson et al., 2002; Yue et al., 2003). These changes extend to the periplasmic side and might be responsible for the receptor's interaction with the TonB complex. Therefore, these allosteric transitions tightly regulate the opening of a putative transient channel or permeation path for the transport of the substrate into the periplasm (Shultis et al., 2006; van der Helm, 2004; van der Helm et al., 2002). As suggested by Shultis et al. (2006) the degree of this allosteric transition will be a function of the size and chemical characteristics of each ferric-siderophore complex.

The experiments delineated in this work explored the effects of mutations in specifically conserved residues on the transport function of FatA. Our molecular modelling and experimental results underscore the fact that although there is a low sequence identity between the known siderophore receptors, the mechanisms of transport will probably share some similarities among them. Moreover, our results also suggest that a conformational change or partial unfolding of the plug domain occurs during ferric-anguibactin transport.

\section{METHODS}

Bacterial cultures. The strains and plasmids used in this work are listed in Tables 1 and 2 respectively. E. coli strains were grown at $37^{\circ} \mathrm{C}$ in Luria-Bertani (LB) broth supplemented with antibiotics as appropriate: kanamycin $50 \mu \mathrm{g} \mathrm{ml}^{-1}$, chloramphenicol $30 \mu \mathrm{g} \mathrm{ml}^{-1}$ and ampicillin $100 \mu \mathrm{g} \mathrm{m}^{-1}$. V. anguillarum strains were grown at $25^{\circ} \mathrm{C}$ in trypticase soy broth with $1 \%(\mathrm{w} / \mathrm{v})$ sodium chloride (TSBS) supplemented with antibiotics as appropriate: kanamycin $50 \mu \mathrm{g} \mathrm{ml}^{-1}$, chloramphenicol $10 \mu \mathrm{g} \mathrm{ml}^{-1}$ and rifampicin $100 \mu \mathrm{g} \mathrm{ml}^{-1}$. Solid media were obtained by adding $1.5 \%(\mathrm{w} / \mathrm{v})$ agar. For iron-restricted growth $V$. anguillarum was grown in chemically defined CM9 minimal medium $\left[60 \mathrm{~g} \mathrm{Na}_{2} \mathrm{HPO}_{4} \mathrm{l}^{-1}, 30 \mathrm{~g}\right.$

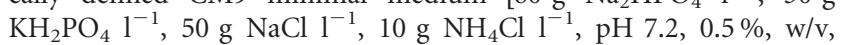
glucose and $0.2 \%$, w/v, Casamino acids (Crosa, 1980)] supplemented with $10 \mu \mathrm{M}$ of the iron chelator ethylenediamine-di-(o-hydroxyphenylacetic) acid (EDDHA). When appropriate IPTG was added to the liquid broth to a final concentration of $1 \mathrm{mM}$. Bacterial growth was monitored by the optical density at $600 \mathrm{~nm}$. Chrome azurol S (CAS) agar plates were used to detect siderophore production (Schwyn \& Neilands, 1987).

General molecular biology procedures. Chromosomal and plasmid DNA purification from $V$. anguillarum and E. coli was performed according to standard protocols (Sambrook et al., 1989). Enzymic digestions and ligations were carried out according to the manufacturer's instructions (New England Biolabs). Total RNA was isolated using the RNAWiz reagent (Ambion), according to the manufacturer's specifications, from $V$. anguillarum 775 and from the CSL-13 cultures grown on CM9 minimal medium. 
Table 1. Bacterial strains

\begin{tabular}{|c|c|c|}
\hline Strain & Relevant characteristics & Source \\
\hline \multicolumn{3}{|c|}{ Escherichia coli } \\
\hline TOP10 & 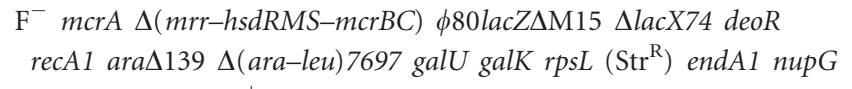 & Invitrogen \\
\hline S17- $\lambda$ pir & thi pro hsdR hsdM ${ }^{+}$recA RP4-2-Tc:: Mu-Km::Tn7 $\lambda$-pir & Simon et al. (1983) \\
\hline MM294 & $\mathrm{F}^{-}$endA1 hsdR17 supE44 thi-1 $\lambda^{-}$harbouring plasmid pRK2013 & Laboratory stock \\
\hline BW25113 & $\begin{array}{l}\Delta(\text { araD-araB }) 567 \Delta \text { lacZ } 4787(:: r r n B 4) \text { lacIp-4000 }\left(\text { lacl } I^{\mathrm{Q}}\right) \lambda^{-} \\
\text {rpoS396(Am) rpH-1 } \Delta(\text { rhaD-rhaB)568 rrnB-4 hsdR514 harbouring } \\
\text { plasmid pKD46 }\end{array}$ & Datsenko \& Wanner (2000) \\
\hline \multicolumn{3}{|c|}{ Vibrio anguillarum } \\
\hline 775 (pJM1) & Wild-type Pacific Ocean prototype & Laboratory stock \\
\hline CSL-13 & $775 \Delta$ fat $A$ & This study \\
\hline CSL-48 & $775 \Delta$ tonB2 & This study \\
\hline CSL-49 & $775 \Delta$ fat $A, \Delta$ tonB2 & This study \\
\hline CSL-50 & $775 \Delta$ fatA, $\Delta$ tonB2, $\Delta$ tonB1 & This study \\
\hline CSL-51 & $775 \Delta$ tonB1 & This study \\
\hline CSL-52 & $775 \Delta$ tonB1, $\Delta$ tonB2 & This study \\
\hline CSL-53 & CSL-13 $\Delta a n g A$ chromosome & This study \\
\hline AC7 & $775 \Delta a n g A$ chromosome & Alice et al. (2005) \\
\hline \multicolumn{3}{|c|}{ Mutants in lock region } \\
\hline CSL-1 & CSL-13/pMMB208 fatA & This study \\
\hline CSL-2 & CSL-13/pMMB208 R95A & This study \\
\hline CSL-3 & CSL-13/pMMB208 R95Q & This study \\
\hline CSL-4 & CSL-13/pMMB208 G96A & This study \\
\hline CSL-5 & CSL-13/pMMB208 E505A & This study \\
\hline CSL-6 & CSL-13/pMMB208 E505Q & This study \\
\hline CSL-7 & CSL-13/pMMB208 E550A & This study \\
\hline CSL-8 & CSL-13/pMMB208 E550Q & This study \\
\hline CSL-9 & CSL-13/pMMB208 K130A & This study \\
\hline CSL-10 & CSL-13/pMMB208 K130Q & This study \\
\hline CSL-11 & CSL-13/pMMB208 F522A & This study \\
\hline \multicolumn{3}{|c|}{ Mutants in channel region } \\
\hline CSL-12 & CSL-13/pMMB208 G131A & This study \\
\hline CSL-14 & CSL-13/pMMB208 G138A & This study \\
\hline CSL-15 & CSL-13/pMMB208 G143A & This study \\
\hline CSL-16 & CSL-13/pMMB208 G131A G138A & This study \\
\hline CSL-17 & CSL-13/pMMB208 G131A G143A & This study \\
\hline CSL-18 & CSL-13/pMMB208 G138A G143A & This study \\
\hline CSL-19 & CSL-13/pMMB208 G131A G138A G143A & This study \\
\hline CSL-20 & CSL-13/pMMB208 R428A & This study \\
\hline CSL-21 & CSL-13/pMMB208 R428E & This study \\
\hline
\end{tabular}

RT-PCR assays. Total RNA was extracted as described above and treated with Turbo DNA-free (Ambion). RT-PCR analysis was performed using the Superscript II reverse transcriptase (Invitrogen) according to the manufacturer's instructions. The PCR reactions were performed as follows: $95^{\circ} \mathrm{C}$ for $5 \mathrm{~min}, 30$ cycles of $95^{\circ} \mathrm{C}$ for $30 \mathrm{~s}, 60^{\circ} \mathrm{C}$ for $60 \mathrm{~s}$ and $72{ }^{\circ} \mathrm{C}$ for $90 \mathrm{~s}$, with a final extension step at $72{ }^{\circ} \mathrm{C}$ for $10 \mathrm{~min}$. The primer used for cDNA synthesis was RR (Table 3 ) and the primers used in the PCR reactions were RR and $\mathrm{RU}$ for the plasmid-encoded $a n g R$ gene; $\mathrm{BR}$ and $\mathrm{BU}$ for the fatB gene; DR and DU for the fatD gene; and A4 and A3 for the fat $\mathrm{A}$ gene (Table 3).

Construction and complementation of the mutants. The $V$. anguillarum fatA mutant strain CSL-13 was constructed using the lambda red system previously described for E. coli (Yu et al., 2000) using the fatADC and fatAUC primers (Table 3) and the pKD4 plasmid (Table 1) as the template. E. coli BW25113 competent cells harbouring the pJHC-T2612 plasmid (Table 1), which contains the iron transport biosynthesis (ITB) operon from V. anguillarum 775 strain, were electroporated with a linear PCR product containing the kanamycin resistance $\left(\mathrm{Km}^{\mathrm{r}}\right)$ cassette flanked by fatA sequences. The $\mathrm{Km}^{\mathrm{r}}$ clones were analysed by sequencing to confirm the site of recombination. The results indicated that in the recombinant pJHC-T2612$\mathrm{C}$ plasmid (Table 2), the fatA gene was replaced by the kanamycin resistance cassette and that only $39 \mathrm{bp}$ and $45 \mathrm{bp}$ from the $5^{\prime}$ and $3^{\prime}$ end respectively of the original gene was present in this construct. This E. coli strain was then mated by triparental conjugation with the wild-type $V$. anguillarum 775 strain using the E. coli strain 
Table 2. Plasmids

\begin{tabular}{|c|c|c|}
\hline Plasmid & Relevant characteristics & Source \\
\hline pDM4 & Suicide plasmid $\mathrm{R} 6 \mathrm{~K}$ origin $\mathrm{Cm}^{\mathrm{R}}$ & Milton et al. (1996) \\
\hline pKD4 & $\mathrm{Km}^{\mathrm{R}}$ & Datsenko \& Wanner (2000) \\
\hline pJHC-T2612 & XhoI partial digest of pJM1 DNA clones in pVK102 & Tolmasky \& Crosa (1984) \\
\hline pJHC-T261-C & fat $A:: \mathrm{Km}$ & This study \\
\hline pCL2 & pCR2.1-wild-type fatA & This study \\
\hline pALE3 & pDM4 harbouring $\triangle a n g A$ chromosome & Alice et al. (2005) \\
\hline
\end{tabular}

MM294, which harbours the pRK2013 helper plasmid (Table 1) to mobilize the recombined product. $\mathrm{Km}^{\mathrm{r}}$ exconjugants were obtained and screened by PCR. The result obtained with the $V$. anguillarum strain CSL-13 indicated that a double recombination between the pJM1 and pJHC-T2612-C plasmids had occurred. This mutant strain was subsequently complemented with a copy of the corresponding wild-type gene as well as with copies of the site-directed mutant genes under the control of the inducible Ptac promoter, using the low copy number plasmid pMMB208 (Table 2) as the vector.

In-frame deletions of the entire coding sequence of the tonB1, tonB2 and angA chromosomal genes were generated using splicing by overlap extension (SOE)-PCR (Senanayake \& Brian, 1995), which resulted in a final product containing less than $10 \%$ of the sequence of the wild-type gene. For the ton $B 1$ gene the primers used for SOE were $1 \mathrm{~B} 1 \mathrm{AU}$, 2B1AUR, 3B1ARU and 4B1AR and for tonB2 primers 1B2AU, 2B2AUR, 3B2ARU and 4B2AR (Table 3). The fragments containing each deletion were cloned in the plasmid pCR2.1-TOPO, then subcloned in the suicide vector pDM4 (Table 2) and transformed into E. coli S17-1 $\lambda$ pir (Table 1). For the construction of the double $\triangle$ fat $\triangle a$ angA mutant strain we used the suicide plasmid pALE3 (Alice et al., 2005). The resulting suicide plasmids were mobilized by conjugation into $V$. anguillarum 775 and CSL-13 using the chloramphenicol resistance for selection. After the conjugation, rifampicin $\left(100 \mu \mathrm{g} \mathrm{ml}^{-1}\right)$ was used for counter-selection against $E$. coli. The exconjugants were screened using a sucrose-resistance-based counter-selection strategy and all the isolated mutants were confirmed by PCR analysis. To serve as a control the pMMB208 vector was conjugated into each strain (Table 2).

Site-directed mutagenesis. The fatA gene was amplified with the primers fatAUP and fatAD (Table 3 ) using the $P f u$-Taq polymerase (Stratagene) and subcloned into the pCR-BluntII vector (Invitrogen). The resulting plasmid, pCL2, was used as a template

Table 3. Primers

\begin{tabular}{|c|c|c|}
\hline Name & Sequence $\left(5^{\prime}-3^{\prime}\right)$ & Used in \\
\hline RU & TTAGCAAGCTACGCCAAGAG & PCR \\
\hline BU & ACACTCCTGACATTTCGGAC & PCR \\
\hline $\mathrm{DR}$ & GAGGCTATATCTTCCСССАT & PCR \\
\hline A3 & GGTCAAAGTCGTAAAGCGCA & PCR \\
\hline fatAUP & CTAGACCAACGTAGTCCAGC & PCR \\
\hline fatAD & AACCAGAAAGGTCAAGGGCA & PCR \\
\hline fatADC & ATCCGCTGTAGCAGATAGCATCACCGTACGAGGGGCACCCCATATGAATATCCTCCTTAG & Lambda Red \\
\hline fatAUC & ATGACTCATCAGGTAGCGACCTGTCATAAAAAACAATCTGTGTAGGCTGGAGCTGCTTCG & Lambda Red \\
\hline $1 \mathrm{~B} 2 \mathrm{AU}$ & GGCAGTAGCGATCCTAAATTGATG & SOE \\
\hline 2B2AUR & TCCACAGTCACGGTTTGTCCCGGCAGCGAAATCGCTCCTGCAATTGG & SOE \\
\hline 3B2ARU & CCAATTGCAGGAGCGATTTCGCTGCCGGGACAAACCGTGACTGTGGA & SOE \\
\hline $4 \mathrm{~B} 2 \mathrm{AR}$ & TAAACCGACCAACTGTCGCCACCA & SOE \\
\hline
\end{tabular}


for the site-directed mutagenesis, which was performed using the QuickChange site-directed mutagenesis kit (Stratagene) according to the manufacturer's instructions. The sequencing reactions were performed at the Vollum Institute Sequencing Core facility, using a 3130xL Genetic Analyser (Applied Biosystems) with the BigDye Terminator v3.1 Cycle Sequencing Ready Reaction Kit (Applied Biosystems) and the samples were run on a $50 \mathrm{~cm}$ capillary array with the POP-7 polymer.

Anguibactin purification. The siderophore purification procedures were carried out using glassware that had been washed with $0.1 \mathrm{M}$ $\mathrm{HCl}$. The temperature during the purification procedure never exceeded $25^{\circ} \mathrm{C}$. Anguibactin was purified as described previously (Actis et al., 1986). The purity of the siderophore was confirmed by HPLC (Beckman Gold HPLC System), monitoring at $264 \mathrm{~nm}$ using a C18 reverse-phase HPLC column (YMC, AQ-312, $150 \times 6 \mathrm{~mm}$ ID), and as mobile phase: solvent $\mathrm{A}\left[\mathrm{H}_{2} \mathrm{O} 0.01 \%\right.$, v/v, trifluoroacetic acid (TFA)] and solvent B (acetonitrile $0.01 \%, v / v$, TFA). The gradient used was $100 \%$ solvent A for $2 \mathrm{~min}$, followed by an increasing concentration of solvent B from 0 to $100 \%$ over $30 \mathrm{~min}$. The biological activity of the purified anguibactin was assessed by bioassays using strain AC7 (Alice et al., 2005), which is siderophore-production-deficient but FatA-receptor-proficient.

${ }^{55}$ Fe-anguibactin uptake. The ${ }^{55} \mathrm{Fe}$-anguibactin complex was prepared using a stock solution of ${ }^{55} \mathrm{FeCl}_{3}$ in $0.5 \mathrm{M} \mathrm{HCl}$ (specific activity $103.46 \mathrm{mCi} \mathrm{mg}^{-1}, 3828 \mathrm{MBq} \mathrm{mg}{ }^{-1}$; Perkin Elmer). The ${ }^{55} \mathrm{FeCl}_{3}$ was diluted in MOPS buffer $(0.1 \mathrm{M} \mathrm{pH} 7.0)$ to a final concentration of $0.8 \mathrm{mM}$ in the presence of $1.2 \mathrm{mM}$ of the iron chelator EDDHA in order to maintain the ferric iron in solution and this complex was then incubated for $10 \mathrm{~min}$ at room temperature. After this incubation period, the deferrated anguibactin $(1 \mathrm{mM})$, also prepared in $0.1 \mathrm{M}$ MOPS pH 7.0, was added. Due to its instability, the ${ }^{55} \mathrm{Fe}-$ anguibactin complex was used fresh or stored for only 3 days at $-80^{\circ} \mathrm{C}$. The cultures used for this assay were grown in CM9 minimal medium supplemented with $1 \mathrm{mM}$ IPTG until exponential phase $\left(\mathrm{OD}_{600} 0.5-0.8\right)$. The cultures were centrifuged and resuspended in CM9 supplemented with $10 \mu \mathrm{M}$ EDDHA in order to chelate the available iron in the medium and induce the ferric-anguibactin transport system as well as the energy transduction system. These cultures were then incubated for $2 \mathrm{~h}$ at $25^{\circ} \mathrm{C}$ in order to starve the cells of internal iron. It is worth mentioning that this EDDHA concentration, which is lower than the minimal inhibitory concentration detected for the $V$. anguillarum 775 strain $(75 \mu \mathrm{M})$, is sufficient to prevent cellular multiplication during the incubation period. After this incubation period the cells were centrifuged and resuspended in half of the original volume of complete minimal medium (without EDDHA). Bacterial dilutions performed in the same medium were used to calculate the colony-forming units (c.f.u.). For the time- and concentration-dependent curves $50 \mathrm{ml}$ tubes containing $20 \mathrm{ml}$ complete minimal medium and the desired ${ }^{55} \mathrm{Fe}$-anguibactin concentration were prepared. For each tube, $100 \mu \mathrm{l}$ of the cellular suspension was added. During the experiment the cells were incubated under agitation at 200 r.p.m. at room temperature. After each incubation period the cellular suspension was quenched by the addition of a 100-fold dose of non-labelled ferricanguibactin, rapidly filtered through a $0.22 \mu \mathrm{m}$ filter (Millipore Corporation) and washed three times with $10 \mathrm{ml} 2 \mathrm{M} \mathrm{LiCl}$. The filters were air-dried and the radioactivity measured in a liquid scintillation counter. The $K_{\mathrm{m}}$ and $V_{\max }$ were calculated using ferricanguibactin concentrations ranging from 0.01 to $75 \mathrm{nM}$. In the case of the G138A G143A double mutant, the concentrations used were from 0.01 to $200 \mathrm{nM}$. Kinetic parameters were determined from the initial rate of ${ }^{55} \mathrm{Fe}$-anguibactin uptake, calculated at different substrate concentration at $20 \mathrm{sec}$ and $1 \mathrm{~min}$ for all the strains. The kinetic parameters $K_{\mathrm{m}}$ and $V_{\max }$ were evaluated and treated as those for an enzyme reaction using the Graphic Pad Prism4 program.
Non-specific binding has been subtracted for the determination of the transport rates and kinetic parameters by using the values obtained for the double $\triangle$ fatA $\Delta$ tonB2 $V$. anguillarum mutant complemented with the wild-type fatA gene. The Scatchard plot shown in Fig. 2 was obtained by linearizing the saturation binding data shown in the same figure using the Graphic Pad Prism4 program.

${ }^{55} \mathrm{Fe}$-anguibactin binding. The ${ }^{55} \mathrm{Fe}$-anguibactin binding assays were performed in a similar way to that described above for the transport assays, with minor modifications. Experiments were carried out in the presence of $1 \mathrm{mM}$ of the respiratory inhibitor $\mathrm{KCN}$ and after the incubation period of $2 \mathrm{~h}$ in the presence of the iron chelator EDDHA, the cells were resuspended in salts minimal medium without carbon source and Casamino acids. For the concentration-dependent curves ${ }^{55} \mathrm{Fe}$-anguibactin concentrations ranging from 0.01 to $750 \mathrm{nM}$ were used. Since we have previously determined that equilibrium was reached after 2 min incubation in the presence of the ${ }^{55} \mathrm{Fe}$-siderophore, this incubation time was used for the binding experiments. The $K_{\mathrm{D}}$ was calculated using Graphic Pad Prism4. The curves shown in Fig. 2 were fitted to one binding site hyperbola using the same software.

Sequence alignments. The sequences of the outer-membrane receptors from different organisms were simultaneously aligned using CLUSTALW (Thompson et al., 1994).

FatA modelling. To obtain a structural model for FatA the structures of the E. coli receptors FhuA (Ferguson et al., 1998; Locher et al., 1998), FepA (Buchanan et al., 1999), BtuB (Chimento et al., 2003) and FecA (Ferguson et al., 2002) and the P. aeruginosa FpvA receptor (Cobessi et al., 2005b) together with the multiple sequence alignment of 29 different ferric-siderophore and haem transporters (van der Helm, 2004) were used. Since the E. coli FhuA receptor displays the highest identity to the $V$. anguillarum FatA receptor $(23 \%$ identity) it was chosen as the platform for the FatA model. In the multiple sequence alignment of 29 proteins in this family, about 25 residues are conserved or identical; half of these residues belong to the plug region of the proteins and the rest are spread over a number of transmembrane $\beta$ strands from strand 11 to strand 22 (van der Helm, 2004). Interestingly, none of the conserved residues occur on extracellular loops with the exception of those at the beginning of loop 11. The conserved residues in the plug region occur at identical locations and orientation in five different crystal structures (see Results and Fig. 3b). These structural features of the plug region and the presence of conserved residues allow the sequence of FatA to be fitted in the structure of this region of FhuA, although it required several small deletions, insertions and a number of mutations. The insertions and deletions required were made in the middle of the extracellular loops using the SPDBV program (Guex \& Peitsch, 1997). Construction of the remainder of the model was adversely affected by the fact that, as previously mentioned, the identity between FatA and FhuA is low and by the observation that extracellular loops in the proteins with known structure vary greatly in length. In addition, for many transmembrane strands the $\beta$ secondary structure extends far above the surface of the outer membrane. However, the multiple sequence alignment together with the structural information from the $\beta$ strands and the location of the conserved residues in the known structures allowed the assignment of the transmembrane $\beta$ strands of the 22 -stranded $\beta$ barrel of FatA, obtaining a better certainty for the latter half of the $\beta$ barrel. The resulting FatA model was refined by molecular dynamics (MD) according to Brunger et al. (1998) and subsequently with energy minimizations using the SPDBV program (Guex \& Peitsch, 1997). After convergence was obtained, the complete water structure of FhuA was added to the FatA model and once more MD and energy 
minimization were applied. The final model did not show unusual intermolecular contacts and none of the bond lengths, bond angles or conformational angles showed unusual energy values.

Isolation of outer-membrane fractions. This was performed as described previously using $1.5 \%(\mathrm{w} / \mathrm{v}) \quad \mathrm{N}$-laurylsarcosine (Sigma) (Crosa \& Hodges, 1981). The cultures used for the outer-membrane purification were grown in CM9 minimal medium supplemented with $1 \mathrm{mM}$ IPTG until exponential phase $\left(\mathrm{OD}_{600} 0.5-0.8\right)$. Protein was quantified using the BCA Protein Assay Kit (Pierce Biotechnology) and BSA as standard. For the densitometry analysis $10 \mu \mathrm{g}$ of the outer-membrane isolated fractions were separated on $12 \%$ SDS-PAGE (Criterion-XT Precast gel, Bio-Rad Laboratories) using XT-MOPS buffer (Bio-Rad Laboratories) and stained with Coomassie blue using standard protocols. The gel images were captured using a Kodak Gel Logic Imaging System and analysed using the Kodak 1D Image Analysis software.

Western blots. For the Western blots $5 \mu \mathrm{g}$ of the outer-membrane isolated fractions were separated on $12 \%$ SDS-PAGE (Criterion-XT Precast gel, Bio-Rad Laboratories) using XT-MOPS buffer (Bio-Rad Laboratories) and transferred for $4 \mathrm{~h}$ at $400 \mathrm{~mA}$ into $0.45 \mu \mathrm{m}$ Immobilon-P membranes (Millipore) using a Trans-Blot Electrophoretic Transfer Cell (Bio-Rad Laboratories). The transfer buffer used was $25 \mathrm{mM}$ Tris, $192 \mathrm{mM}$ glycine and $20 \%(\mathrm{v} / \mathrm{v})$, methanol, $\mathrm{pH}$ 8.3. After transfer the membranes were blocked overnight at $4{ }^{\circ} \mathrm{C}$ using $10 \%(\mathrm{w} / \mathrm{v})$, BSA in TBST buffer $(20 \mathrm{mM}$ Tris $\mathrm{pH} 7.6,0.3 \mathrm{M} \mathrm{NaCl}$ and $0.05 \%, \mathrm{v} / \mathrm{v}$, Tween-20). The membranes were washed four times with TBST buffer and then incubated for $1 \mathrm{~h}$ with anti-FatA polyclonal antibody diluted 1/15000 in TBST $5 \%(\mathrm{w} / \mathrm{v})$, BSA (TBST-BSA). After this incubation the membranes were washed four times with TBST buffer and then incubated for $1 \mathrm{~h}$ using as secondary antibody goat anti-rabbit $\operatorname{IgG},[\mathrm{H}+\mathrm{L}$, horseradish peroxidase (HRP) conjugated (Pierce Biotechnology) diluted $1 / 20000$ in TBST-BSA. The membranes were washed four times with TBST buffer and then incubated for $5 \mathrm{~min}$ with the Immobilon Western Chemiluminiscent HRP substrate (Millipore). The Westerns were developed using Kodak BioMax MR film and the images captured and analysed as described above.

\section{RESULTS}

\section{Construction and characterization of a $\mathbf{V}$. anguillarum fat $A$ mutant strain}

To understand the mechanism of transport mediated by FatA we needed to construct a fatA null mutant to use as a recipient in complementation experiments. However, because the fatA gene is part of the iron-transport biosynthesis (ITB) operon it was required that this mutant was non-polar (Fig. 1a). The absence of polar effects due to the replacement of the fatA gene with an antibiotic resistance cassette was assessed by RT-PCR (Fig. 1a). The results clearly showed that the replacement of the fat $A$ gene by a kanamycin resistance cassette did not affect the expression of downstream genes, such as the positive regulator and biosynthesis gene angR (Wertheimer et al., 1999). The expression of the upstream genes $f a t B$ and fat $D$ was also confirmed by RT-PCR (Fig. 1a). As expected from a receptor with no $\mathrm{N}$-terminal regulatory domain, such as those present in FecA in E. coli and FpvA in P. aeruginosa, the absence of the outer-membrane protein receptor FatA in $V$. anguillarum caused a significant overproduction of the siderophore anguibactin, as assessed by using chrome azurol S (CAS) medium (data not shown). The null mutation was also confirmed by Western blot analysis. Fig. 1(b) shows the amount of receptor produced in the $\Delta f a t A$ mutant complemented with the wild-type gene (lane 2) as compared to that expressed from the pJM1 indigenous plasmid (lane 1). Also shown is the result obtained with the $\Delta f a t A$ mutant harbouring the empty vector pMMB208 (lane 3). Densitometric analysis indicated that in the mutant complemented with the wild-type gene, (a)
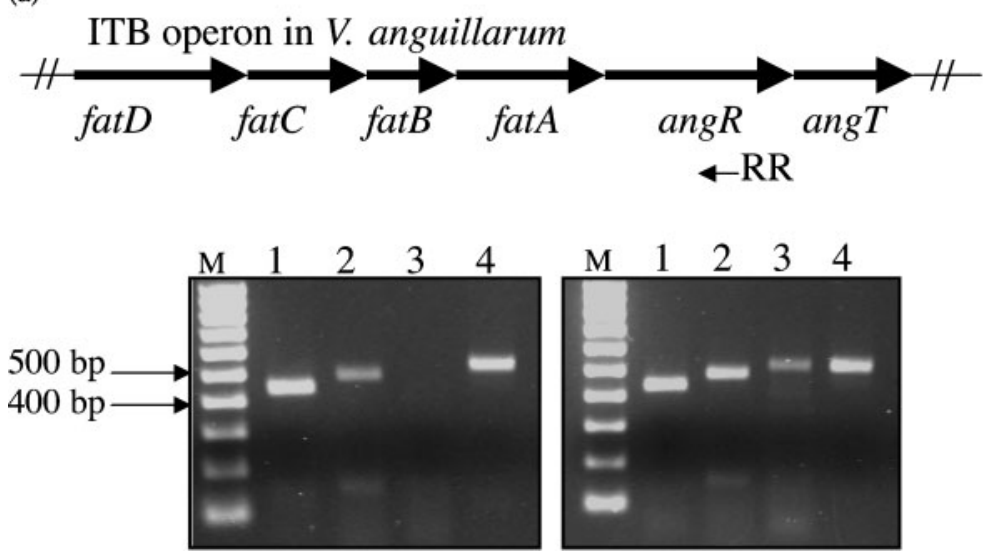

(b)

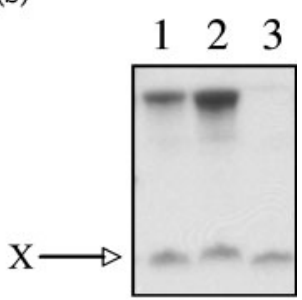

Fig. 1. Characterization of a $V$. anguillarum $\Delta$ fat $A$ mutant. (a) Scheme of the ITB operon in $V$. anguillarum and RT-PCR analysis of RNA extracted from the $V$. anguillarum 775 wild-type (right panel) and the $\triangle$ fat $A$ mutant strain (left panel). The reverse transcription reactions were carried out using a primer complementary to the ang $R$ gene (RR) and the PCR reactions with primers complementary to the fatD (lanes 1), fatB (lanes 2), fat $A$ (lanes 3 ) and ang $R$ (lanes 4) genes as indicated in Methods. M, low molecular mass marker. (b) Western blot using FatA antiserum of the wild-type V. anguillarum 775 (lane 1), the single $\triangle$ fatA mutant complemented with the wild-type fatA gene (lane 2), and the empty vector pMMB208 (lane 3). X denotes a non-specific cross-reacting band. 


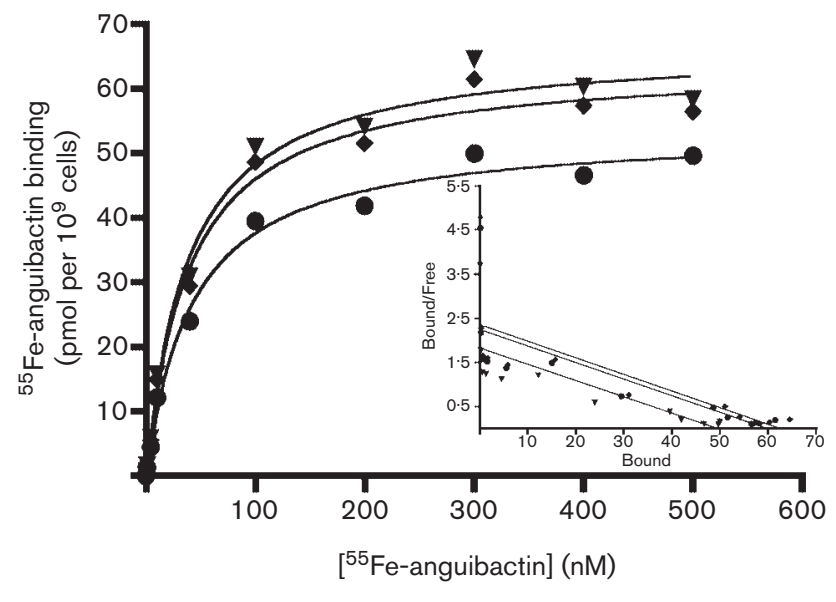

Fig. 2. Binding of ${ }^{55} \mathrm{Fe}$-anguibactin. Determination of binding to FatA in the presence of $1 \mathrm{mM}$ potassium cyanide $(\boldsymbol{\nabla})$ or $1 \mathrm{mM}$ dinitrophenol (O) as respiratory inhibitors. The $\Delta$ tonB2 mutant strain ( $)$ was used as a control without respiratory inhibitors. The Scatchard plot of the results is shown in the inset.

receptor expression is at least twofold higher than in the wild-type 775 (Fig. 1b). To quantify this difference we used the nonspecific band denoted as $\mathrm{X}$ that cross-reacts with the FatA antiserum (Fig. 1b) as a loading control. Further comparisons of the amounts of FatA in the site-directed mutants were carried out using the complemented strain as a control.

\section{${ }^{55} \mathrm{Fe}$-anguibactin binding in $\mathbf{V}$. anguillarum}

To determine the binding of ${ }^{55} \mathrm{Fe}$-anguibactin to the FatA receptor we used the respiratory inhibitor $\mathrm{KCN}$ and the membrane uncoupler dinitrophenol (DNP). In our studies we were not able to perform the binding experiments at $0^{\circ} \mathrm{C}$ as routinely performed in E. coli (Chakraborty et al., 2003), P. aeruginosa (Shen et al., 2005) and Listeria monocytogenes (Jin et al. 2006) because at low temperatures lysis of V. anguillarum cells occurs. In addition, as a control we also used the $\Delta$ tonB2 mutant strain, in which transduction of the proton-motive force to the outer-membrane receptor FatA is abolished. We observed similar effects for both respiratory poisons as compared with the $\triangle$ tonB2 mutant strain, indicating that under the conditions tested no energy was being transduced through TonB2 (Fig. 2). Therefore, we decided to characterize the dissociation constant $\left(K_{\mathrm{D}}\right)$ of the FatA receptor in the presence of the respiratory inhibitor KCN.

The results indicated that FatA has a $K_{\mathrm{D}}$ of $26 \pm 3 \mathrm{nM}$ for the complex with ferric-anguibactin. To assess whether the TonB2 protein plays a role in the binding of the ferricanguibactin complex to FatA, we also determined the $K_{\mathrm{D}}$ value for the FatA receptor in the absence of this energytransducing protein. The $K_{\mathrm{D}}$ values obtained for FatA in a $\Delta$ ton $B 2$ background determined in the presence and absence of $\mathrm{KCN}, 29 \pm 3 \mathrm{nM}$ and $23 \pm 1 \mathrm{nM}$ respectively, indicated that $\mathrm{TonB} 2$ is not essential for the binding process.

\section{FatA modelling}

The FatA model shown in Fig. 3a is based on the FhuA structure as described in the Methods (Ferguson et al., 1998; (a)

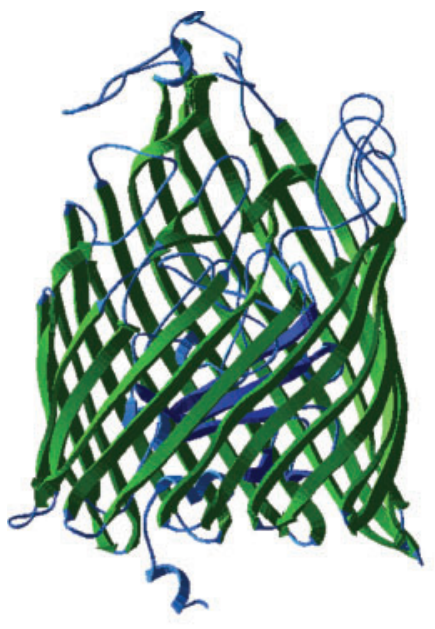

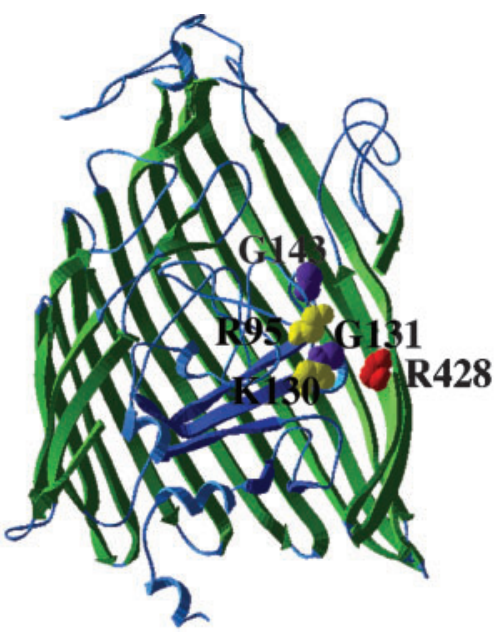

(b)
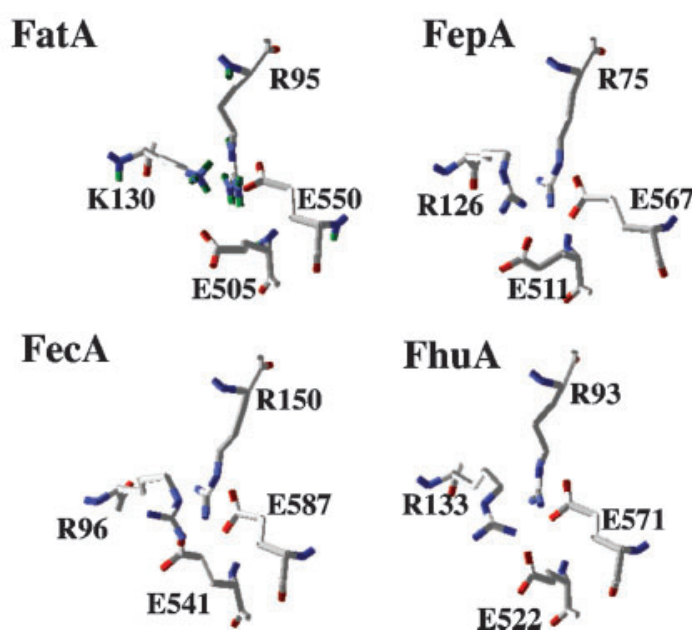

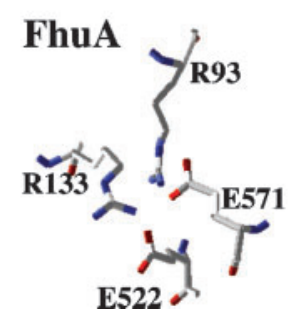

Fig. 3. Molecular modelling of FatA. (a) Representation of the $\beta$ barrel model of FatA viewed from one side. For clarity some of the $\beta$ strands have been removed from the left figure to show the plug domain of FatA, which is shown in blue. The yellow spheres in the structure represent two residues of the 'lock region' R95 and K130, the purple spheres two conserved glycines of the 'channel region' G131 and G143, and the red one the conserved arginine R428 in strand 11. (b) Representation of the conserved amino acid cluster of the quadrupole in the $V$. anguillarum outer-membrane receptor Fat $A$ and in the $E$. coli outermembrane receptors FepA, FecA and FhuA. 


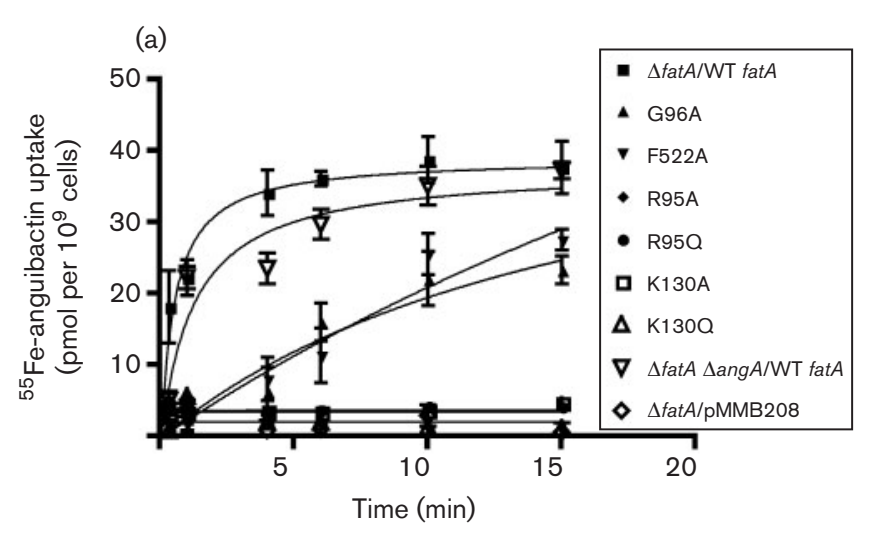

(b)

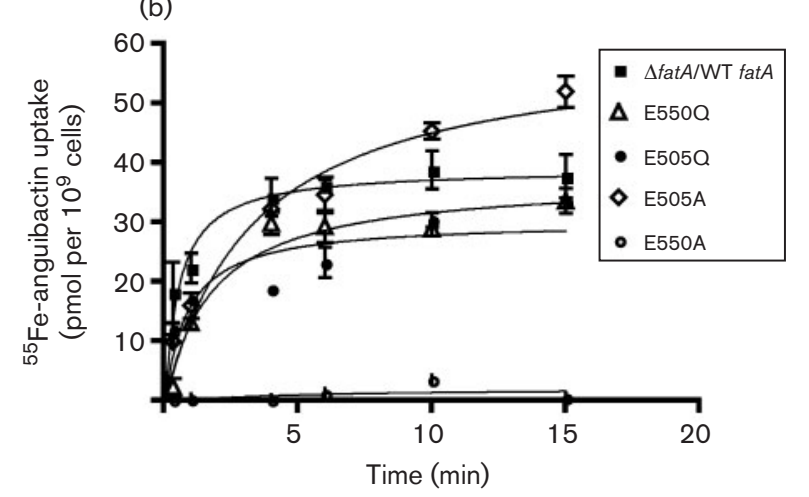

(c)

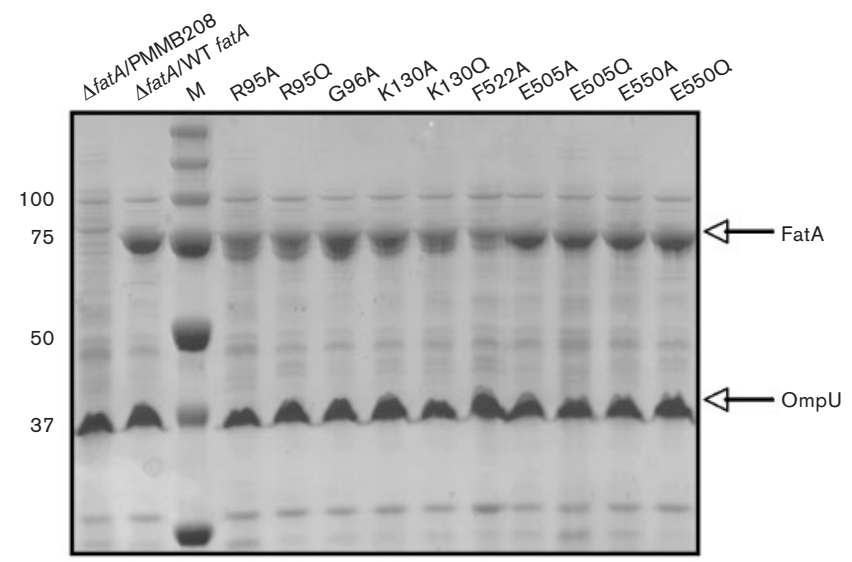

Fig. 4. $(a, b){ }^{55} \mathrm{Fe}$-anguibactin uptake kinetics of the single $\triangle$ fat $A$ mutant, double $\triangle$ fat $A \Delta a n g A$ mutant and the FatA sitedirected mutants of the quadrupole region. The values represent the mean \pm SD of at least three independent experiments. The ${ }^{55} \mathrm{Fe}$-anguibactin concentration used was $10 \mathrm{nM}$. (c) SDS-PAGE of isolated outer-membrane fractions of the FatA mutants. Locations of FatA and $\mathrm{OmpU}$ are denoted with arrows. $\mathrm{M}$, molecular size markers.

Locher et al., 1998). The model shows the well-known plug region (Fig. 3a, b) with the central 4 -stranded $\beta$ sheet and the 22-stranded $\beta$ barrel (Fig. 3a). This model was developed from a set of 25 strictly conserved and identical residues identified using multiple sequence alignment, together with the observed identical locations of these residues within the crystal structures of FepA, FhuA, FecA, $\mathrm{BtuB}$ and FpvA. It is notable that all the conserved residues are located below the A, B and C apices, the binding sites of the ferric siderophores, and are therefore not involved in the primary binding of these substrates (van der Helm, 2004). The presence of several conserved residues within the plug region, named the 'lock region', and its $\beta$ strand structure observed in the crystals, allowed us to design a reliable structure for this region in the FatA model (Fig. 3a, b). However, there are no strictly conserved residues in the multiple sequence alignment or structurally conserved residues in the first 10 transmembrane strands. Furthermore, no conserved residues are found in the extracellular loops; the assignment of the $\beta$ strands $1-9$ in FatA was derived from their position in the known crystal structures delineated in the multiple sequence alignment. Thus, their locations are to some extent uncertain, especially taking into account the different lengths of the extracellular loops in the various structures. Therefore, the model will not be used to analyse the region of the substrate primary binding site on the extracellular loops. Fortunately, due to the presence of strictly conserved residues, this uncertainty does not occur for strands 10-22, which are also structurally conserved in the known crystal structures. The point mutations described in this paper involved only the conserved and identical residues of the plug region and strands 11,14 and 16 of the $\beta$ barrel of FatA. The conserved residues were also the basis for the development of the FatA model and one can therefore expect them to be in the correct position and show the proper interactions with other conserved residues. As ferric-siderophore transport is a process that should be common to all the proteins in this family, our hypothesis is that these conserved residues are involved in the TonB2-dependent transport function of the FatA protein.

\section{${ }^{55} \mathrm{Fe}$-anguibactin transport in $\boldsymbol{V}$. anguillarum}

The investigations of ${ }^{55} \mathrm{Fe}$-anguibactin uptake transport performed with the $\Delta f a t A$ mutant indicated that FatA is the only outer-membrane receptor able to transport anguibactin in V. anguillarum 775 (Fig. 4a). As controls in this study, 
we used the wild-type 775 strain together with the single and double $\triangle$ tonB1 and $\Delta$ tonB2 mutant strains and the results obtained confirmed that anguibactin transport is mediated by the TonB2 system (data not shown), as it was previously described in our laboratory by using bioassays (Stork et al., 2004). Additionally, we have characterized the transport properties of a $V$. anguillarum $\Delta$ fat $A$ mutant unable to produce anguibactin. Since, as we have previously described, the chromosomally encoded angA gene is essential for anguibactin biosynthesis, we constructed the double $\Delta$ fat $A$ $\triangle a n g A$ mutant strain (Alice et al., 2005). The results obtained with this mutant (Fig. 4a) indicated that the de novo synthesis of anguibactin does not affect the interpretation of the experiments described in the following sections. These results were confirmed even at the lowest ${ }^{55} \mathrm{Fe}$-anguibactin concentration used in the transport and binding assays (data not shown).

\section{Site-directed mutations in the 'lock region' of FatA}

In the case of the E. coli receptor FepA, the N-terminal and barrel domains contain a cluster of ten residues named the 'lock region' (Chakraborty et al., 2003). Four of these conserved residues form the quadrupole that consists of amino acids R75, located at the end of strand $\beta 3$; E511, located in strand 14 of the $\beta$-barrel; E567, in strand 16 of the $\beta$-barrel; and R126, located at the end of strand 5 of the mixed $\beta$ sheet (Chakraborty et al., 2003, and Fig. 3b). These residues form hydrogen bonds with each other and are structurally conserved in location, orientation and bonding pattern in the E. coli receptors FecA, FepA and FhuA (Fig. $3 \mathrm{~b}$ ). This is also the case with the E. coli vitamin B12 transporter BtuB (Chimento et al., 2003). Moreover, other homologous residues support this cluster, such as serine or aspartate, proline, phenylalanine or tyrosine and also glycines. The conserved residues in the quadrupole form two separate points of contact between the plug (globular) domain, which keep the plug domain in place within the $\beta$ barrel.

The FatA model described above, together with the sequence alignments suggest that some of the residues belonging to the 'lock region' in FatA consist of the charged amino acid residues R95, K130, E505 and E550 (Fig. 3b). Therefore, these residues were mutated to alanine and to glutamine in order to determine both the transport kinetics and the binding parameters of ferric-anguibactin. We determined the latter by means of dissociation constants of the wild-type and each site-directed mutant. Characterization of transport kinetics of the wild-type FatA indicated that this receptor transports ${ }^{55} \mathrm{Fe}$-anguibactin with a $K_{\mathrm{m}}$ of $8 \mathrm{nM}$ and a $V_{\max }$

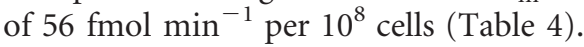

All the mutants showed similar $K_{\mathrm{D}}$ values within the standard deviation range as compared to the wild-type receptor, confirming that the mutations present in the FatA variant proteins did not affect the binding of ferricanguibactin (Table 4). Our results showed two groups of mutants, those in which ferric-anguibactin is still transported, including E505A, E505Q, E550Q, F522A and G96A (Fig. 4a, 4b) and those in which transport is abolished, as in R95A, R95Q, K130A, K130Q and E550A mutants (Fig. 4a, $4 \mathrm{~b})$. We decided to determine the kinetic constants $\left(K_{\mathrm{m}}\right.$ and $V_{\max }$ ) of the mutants able to transport ferric-anguibactin as described in Methods.

The expression of the FatA variant proteins shown in Fig. 4(c) indicated that in all of the site-directed mutants the

Table 4. Kinetic parameters of site-directed mutants located in the FatA 'lock region'

The $K_{\mathrm{D}}, K_{\mathrm{m}}$ and $V_{\max }$ values were calculated using the Graph Pad Prism4 program as described in Methods and they represent the mean \pm SD of at least three independent experiments.

\begin{tabular}{|c|c|c|c|}
\hline Mutations in the lock region ${ }^{\star}$ & $K_{\mathrm{D}}(\mathrm{nM}) \dagger$ & $K_{\mathrm{m}}(\mathrm{nM})$ & $\begin{array}{c}V_{\max }\left(\mathrm{fmol} \mathrm{min} \mathrm{min}^{-1}\right. \\
\left.\text { per } 10^{8} \text { cells }\right)\end{array}$ \\
\hline$\Delta$ fat $A$ /wild-type fat $A$ & $26 \pm 3$ & $8 \pm 1$ & $56 \pm 4$ \\
\hline R95Q & $23 \pm 2$ & NA & NA \\
\hline R95A & $18 \pm 4$ & NA & NA \\
\hline G96A & $40 \pm 5$ & $6 \pm 1$ & $9 \pm 1$ \\
\hline K130A & $24 \pm 6$ & NA & NA \\
\hline K130Q & $36 \pm 6$ & NA & NA \\
\hline E505A & $28 \pm 3$ & $12 \pm 5$ & $54 \pm 6$ \\
\hline E505Q & $18 \pm 3$ & $5 \pm 2$ & $20 \pm 3$ \\
\hline E550A & $32 \pm 5$ & NA & NA \\
\hline E550Q & $32 \pm 1$ & $7 \pm 2$ & $37 \pm 4$ \\
\hline F522A & $20 \pm 7$ & $13 \pm 2$ & $15 \pm 1$ \\
\hline
\end{tabular}

NA, Not applicable.

${ }^{*}$ The numbers represent the position of the mutated residue in the FatA sequence.

$\dagger$ The $K_{\mathrm{D}}$ values were determined in the presence of $1 \mathrm{mM} \mathrm{KCN}$. 
receptor is located in the outer membrane. Densitometry analysis of the gels indicated that the mutants synthesized the receptor in similar amounts as compared to the wildtype (Fig. 4c). We only detected lower amounts of FatA in the R95Q and F522A receptor mutants, with the levels of these variant receptors twofold lower as compared to the wild-type as determined by analysing outer-membrane fractions of three independent cultures. These results confirmed that the absence of transport in several of the mutants is not due to a problem in the expression of the proteins but to their structural characteristics. Curiously, for the R95A, R95Q, G96A, K130A, K130Q and F522A mutants we observed two bands at the FatA location (Fig. 4c). The densitometric analyses mentioned above were performed with the higher molecular mass band; however, these results suggest that there might be increased FatA degradation in these mutants because the lower molecular mass band also showed cross-reactivity with the FatA antisera (data not shown). The $K_{\mathrm{D}}$ values shown in Table 4 indicate that this degradation product does not affect the proper binding of the ferric-siderophore complex.

The FatA variant receptor in which the conserved glycine G96 was replaced with alanine showed similar $K_{\mathrm{m}}$ values as compared to the wild-type receptor; however, there is a sixfold decrease in the $V_{\max }$ of this variant receptor (Table 4 , Fig. 4a). The F522 residue, which might support the side chain of the R95 residue, when replaced with alanine resulted in variant protein with lower transport rates as compared to that of the wild-type (Fig. 4a) but a similar $K_{\mathrm{m}}$ value (Table 4). These results suggest that although these two residues are supporting the quadrupole cluster they are not indispensable for ferric-anguibactin transport.

The R95 residue in FatA will form salt bridges with the glutamic acid residues at position E505 and E550, while the K130 will form a salt bridge with the E550 (Fig. 3b). The substitutions of these glutamic acids by glutamines should allow the formation of hydrogen bonds instead of the salt bridges previously mentioned. However, an alanine substitution will not even allow the formation of a hydrogen bond. Our results indicate that a change in the basic group at position 95 by a polar but uncharged glutamine or by a nonpolar aliphatic residue such as alanine affects the points of interaction between the globular domain and the $\beta$ barrel, without affecting the binding properties of the transporter (Fig. 4a, Table 4).

The sequence alignment of various siderophore receptors showed another conserved arginine in the quadrupole (data not shown). This residue can also be a lysine in some receptors, such as K130 in FatA. One significant feature about the FatA quadrupole is that mutations in this basic group, either to alanine or to glutamine, resulted in a complete loss of transport, suggesting that this residue, as is the case for the R95, is also essential for the proper interaction of the plug domain with the $\beta$ barrel. Table 4 shows that the binding properties of the K130A and K130Q variant proteins were similar to that of the wild-type strain.
In the same vein, substitution of the glutamic acid at position 550 by alanine also affects this region, not allowing the formation of hydrogen bonds and preventing ferricanguibactin transport (Fig. 4b). However, its replacement by glutamine resulted in a FatA variant receptor that had wild-type $K_{\mathrm{m}}$ and $V_{\max }$ values (Table 4 ). The $\mathrm{K}_{\mathrm{m}}$ and $V_{\max }$ values of the mutated E505A receptor were similar to those of the wild-type (Table 4). In the case of the E505Q receptor mutant, the $K_{\mathrm{m}}$ showed similar values as compared to the wild-type receptor; however, we observed a twofold decrease in the $V_{\max }$ value (Table 4). The results obtained with these mutants are in agreement with our hypothesis in which the residue E550 most probably forms bonds with both the R95 and $\mathrm{K} 130$ residues.

\section{Site-directed mutations in the 'channel region' of FatA}

In addition to the mutations of residues located in the lock region' of FatA, we also performed site-directed mutagenesis in several conserved glycines in a specific region we called the 'channel region' (Fig. 5a). We hypothesize that the $\beta 5$ $\beta 6$ loop of the plug needs to change its conformation to allow the ferric-siderophore to move from the apices towards the periplasm (Fig. 5b). We propose that these conserved glycines allow this conformational change to occur. Therefore, a glycine to alanine substitution would make this structural rearrangement more difficult or even impossible, leading to a decrease or inhibition of anguibactin transport.

The structures of several siderophore transporters showed that in the $\beta 5-\beta 6$ loop of the plug domain conserved glycines are found at positions i (G131 in FatA) and i+7 (G138 in FatA), which not only aligned perfectly with the sequences of several receptors for ferric siderophores, but also with the transferrin-binding protein TbpA, the lactoferrin receptor LbpA and the haemophore transporter HasR among others (Fig. 5a and van der Helm, 2004). The proposed structural movement would then facilitate the opening of a second gate involving the 'lock region'. Therefore, although a substitution of the conserved glycines by alanines would result in small local changes in the structure, it would probably obstruct conformational changes around the residues. In our study the conserved glycines located in the $\beta 5-\beta 6$ loop of FatA, G131 (i), G138 (i+7) and G143 were mutated to alanine. In order to evaluate the contribution of each conserved glycine, we substituted the G131, G138 and G143 residues by alanines independently and also constructed the three double mutants and one triple mutant.

Most of the variant receptors of the 'channel region' showed similar $K_{\mathrm{D}}$ values within the standard deviation range as compared to the wild-type, indicating that the mutations did not affect the binding of ferric-anguibactin (Table 5). We only detected an increased $K_{\mathrm{D}}$ value for the single G138A mutant and the double G138A G143A mutant strain (Table 5). 
(a)

\begin{tabular}{|c|c|c|c|c|c|c|c|}
\hline & \multicolumn{2}{|c|}{$\begin{array}{l}\text { Strand } 4 \\
\text { Plug }\end{array}$} & $\begin{array}{c}\text { Strand } 5 \\
\text { Plug }\end{array}$ & $\begin{array}{l}\text { B 5-6 Loop } \\
\text { Plug }\end{array}$ & & $\begin{array}{c}\text { Strand } 11 \\
\text { B Barrel }\end{array}$ & \\
\hline pA & L- & 88 & ERIEVLRGI & ARYGN---GA-AGGVVNIITK & 147 & TIVTPGLRFD & 432 \\
\hline EeA & L-VDG & 91 & ERIEVIRG: & AAARYGN---GA-AGGVVNIITK & 150 & TMLTPGLRWD & 430 \\
\hline EeA & L-IDG & 96 & RIEVIRGI & AAARYGS---GA-MGGVVNIITK & 155 & TMLTP & 429 \\
\hline pva & FQYDG & 179 & DRVEV & ATGLLTGA---GS-LGATINLIRK & 129 & LNLFLGGRVV & 509 \\
\hline upB & YEVDG & 174 & DRVEIVRG $I$ & ATGLISGM---GN-PSATINLIRK & 119 & LSLILGSRLI & 510 \\
\hline upA & YQFDG & 170 & DRIEIV & ATGLMTGA---GD-PSAVVNVIRK & 220 & LHLII & 509 \\
\hline huE & YMVDG & 89 & ERVEV & ATGLMTGT---GN-PSAAINMVRK & 238 & LHLI & 440 \\
\hline pta & FELDG & 88 & ERVEILRGS & SNGLLHGT---GN-PAATVNLVRK & 134 & LTLV & 422 \\
\hline CuA & ISFGG & 122 & ERVEVFKG & ANAFINGISPSGSGVGGMINLEPK & 169 & VSLM & 456 \\
\hline huA & NYLNG & 95 & ERAE IMRGI & PSVLYGK--SS-PGGLLNMVSK & 141 & SRYD & 454 \\
\hline XXA & TFLDG & 88 & ERIDVIKGI & SSALYGQ---SI-PGGVVMMTSK & 136 & WHLN & 426 \\
\hline קUA & LYVDG & 92 & QSVELLRG: & QGTLYGK---SA-QGGIINIVTQ & 135 & FDIG & 381 \\
\hline LuA & ASFAG & 91 & $2 Q V E I$ & QSYIQGR---NT-SAGAIVMKSN & 147 & INVN & 383 \\
\hline $\mathrm{CA}$ & VLMDG & 167 & DAID & GGAVRYGP---QS-VGGVVNFVTR & 128 & WTIT & 469 \\
\hline LeB & VLLDG & 186 & ERVEVLRGS & SSAIYGG----GP-TGGIVSITTR & 244 & WSTE & 517 \\
\hline atA & VLVDG & 72 & BHIEV & ATS-LYGG---GS-TGGLINIVTK & 119 & FTLN & 410 \\
\hline amA & ILLDG & 95 & SSIDVMRG & ATSVRYGP---QN-VGGVINFVTK & 146 & WTVIPGLRYE & 406 \\
\hline Tbpa & LTVDG & 102 & KAVEISKGS & SNSVEQGS---GA-LAGSVAFQTK & 163 & ADVGAGLRYD & 581 \\
\hline LpbA & VSVDG & 94 & STVEIDKG & AGSSDHGS---GA-LGGAVAFRTK & 155 & FDFSLGGRYD & 608 \\
\hline HasR & MNIDG & 187 & SGVTIDKG? & ITGGMGSA---GT-LGGIATFNTV & 239 & -LAKDGQRYT & 540 \\
\hline tuB & VLIDG & 83 & QRVEYIRGI & RSAVYGS---DA-IGGVVNIITT & 133 & FTFEGAARSD & 360 \\
\hline emR & TLVDG & 92 & KRVEIVRG & SALLYGS---GA-LGGVISYETV & 140 & VSILAGTRY & 378 \\
\hline & VMFN & 07 & RIDVLKGP & ASLLNGMPPNGS-VGGSINLVTK & 153 & QVTLGLRERQ & 430 \\
\hline
\end{tabular}

(b)

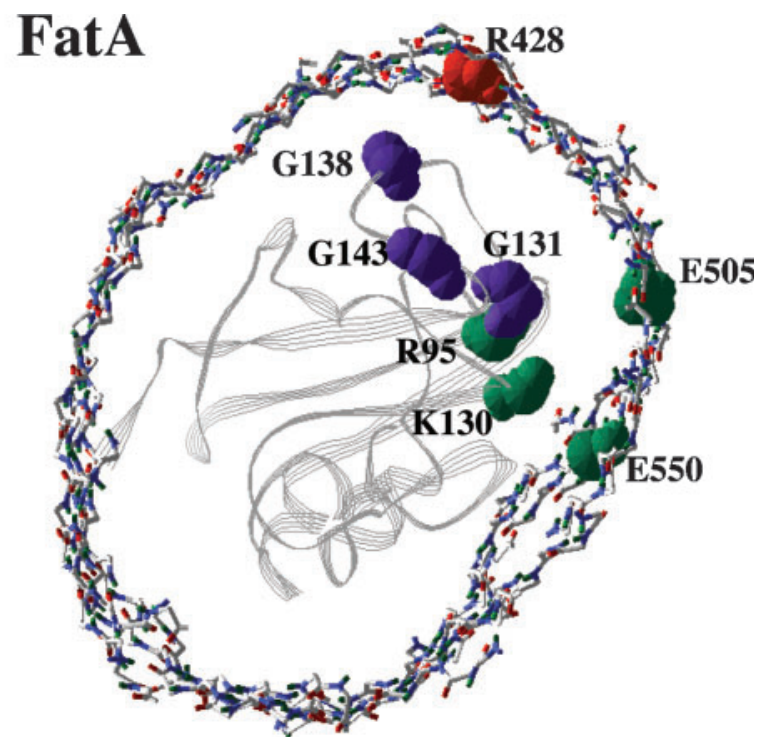

Fig. 5. The 'channel region' of FatA. (a) Sequence alignment of part of the sequences harbouring the 'channel region' of 23 different outer-membrane receptor proteins. The conserved glycines are indicated in bold and the conserved arginine located in strand 11 of the $\beta$ barrel is in underlined bold. (b) Schematic representation of the $\beta$ barrel of FatA viewed from the top. The barrel is indicated in white and for clarity the extracellular loops have been removed from the figure. The plug structure is shown with secondary structure. The green spheres represent the four residues of the 'lock region', the purple spheres the conserved glycines of the 'channel region' and the red sphere the conserved arginine located in strand 11.

The expression levels of most of the variant receptors within the 'channel region' were similar to that of the wild-type as determined by densitometric analysis (data not shown). We only detected higher levels of the G131A G138A variant receptor as compared to the wild-type FatA (data not shown).
Although the single G131A variant receptor showed slower transport rates, after $15 \mathrm{~min}$ of incubation the accumulated ${ }^{55} \mathrm{Fe}$-anguibactin complex reached similar values within the cells to that of the wild-type (Fig. 6a). Moreover, this mutant showed a fivefold increase in the $K_{\mathrm{m}}$ value and a 10 fold decrease in the $V_{\max }$ (Table 5). This defect in transport

Table 5. Kinetic parameters of site-directed mutants located in the FatA 'channel region'

The $\mathrm{K}_{\mathrm{D}}, \mathrm{K}_{\mathrm{m}}$ and $V_{\max }$ values were calculated using the Graph Pad Prism4 program as described in Methods and they represent the mean \pm SD of at least three independent experiments.

\begin{tabular}{|lccc|}
\hline $\begin{array}{l}\text { Conserved glycines and arginine } \\
\text { in the channel region* }\end{array}$ & $\boldsymbol{K}_{\mathbf{D}}(\mathbf{n M}) \dagger$ & $\boldsymbol{K}_{\mathbf{m}}(\mathbf{n M})$ & $\begin{array}{c}\boldsymbol{V}_{\text {max }}\left(\mathbf{f m o l} \mathbf{m i n}^{-\mathbf{1}}\right. \\
\left.\text { per } \mathbf{1 0}^{\mathbf{8}} \text { cells }\right)\end{array}$ \\
\hline$\Delta$ fatA/wild-type fatA & $26 \pm 3$ & $8 \pm 1$ & $56 \pm 4$ \\
G131A & $18 \pm 2$ & $37 \pm 3$ & $6 \pm 0.3$ \\
G138A & $42 \pm 4$ & $22 \pm 6$ & $27 \pm 8$ \\
G143A & $38 \pm 8$ & $27 \pm 5$ & $58 \pm 3$ \\
G131A, G138A & $37 \pm 9$ & $34 \pm 6$ & $23 \pm 5$ \\
G131A, G143A & $20 \pm 2$ & $\mathrm{NA}$ & $\mathrm{NA}$ \\
G138, G143A & $49 \pm 4$ & $54 \pm 7$ & $73 \pm 7$ \\
G131A, G138A, G143A & $30 \pm 2$ & $\mathrm{NA}$ & $\mathrm{NA}$ \\
R428A & $85 \pm 11$ & $\mathrm{NA}$ & $\mathrm{NA}$ \\
R428E & $24 \pm 5$ & $\mathrm{NA}$ & $\mathrm{NA}$ \\
\hline
\end{tabular}

NA, Not applicable.

${ }^{*}$ The numbers represent the position of the mutated residue in the FatA sequence.

$\dagger$ The $K_{\mathrm{D}}$ values were determined in the presence of $1 \mathrm{mM} \mathrm{KCN}$. 
(a)
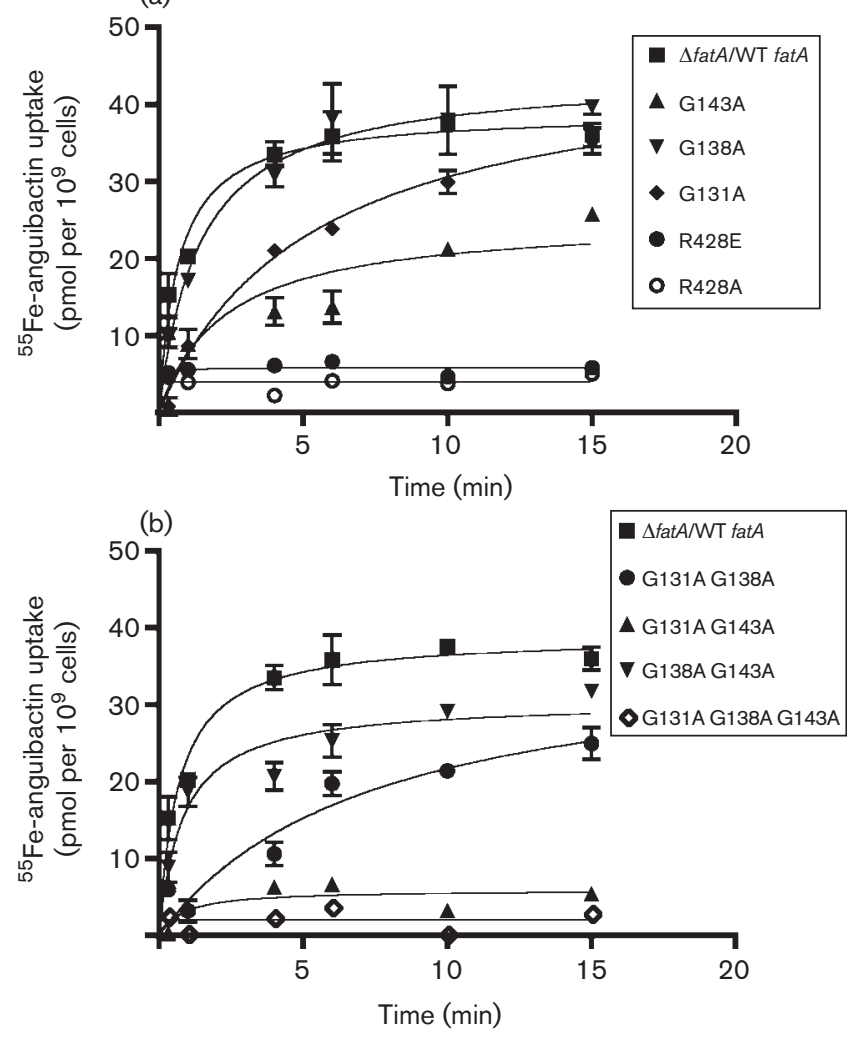

Fig. 6. ${ }^{55} \mathrm{Fe}$-anguibactin uptake kinetics of FatA site-directed mutants. (a) Single mutants of the 'channel region'. (b) Double and triple site-directed mutants of the 'channel region'. The values represent the mean \pm SD of at least three independent experiments. The ${ }^{55} \mathrm{Fe}$-anguibactin concentration used was $10 \mathrm{nM}$. is expected because this residue is adjacent to residue K130, an essential component of the quadrupole as stated in the previous section. The single G138A variant receptor showed similar transport rates to the wild-type (Fig. 6a). However, the $K_{\mathrm{m}}$ value is threefold higher as compared to the wildtype receptor. The lower $V_{\max }$ detected for this receptor suggested that its transport efficiency was lower than that of the wild-type receptor (Table 5). In the case of the single G143A mutant, we observed a similar transport rate to the wild-type; however the $K_{\mathrm{m}}$ value determined is threefold higher (Table 5).

Concerning the behaviour of the double mutants, we observed that the double G131A G143A mutant showed completely defective transport, indicating that these two glycines are essential for the opening of the proposed channel (Fig. 6b). The double G131A G138A and G138A G143A mutant strains were still able to transport ferricanguibactin; however they showed increased $K_{\mathrm{m}}$ values (Table 5, Fig. 6b). As expected, in the triple G131A G138A G143A mutant ferric-anguibactin transport was abolished, confirming the importance of these conserved glycines for ferric-anguibactin transport (Fig. 6b).

It could also be possible that a conserved arginine located on strand 11 of the barrel (R428 in FatA), which is also conserved in all the structures, might be part of this putative 'channel region' (Fig. 5a, b). In the case of this conserved residue, we replaced it with both alanine and glutamic acid.
Our results indicated that when this basic residue is changed to a non-polar aliphatic residue such as alanine or to a residue with an opposite charge, such as glutamic acid, transport of the ferric-siderophore is abolished (Fig. 6a), indicating the importance of this arginine residue during the transport process.

\section{DISCUSSION}

In this work we characterized the transport properties of site-directed mutants in the outer-membrane receptor FatA of $V$. anguillarum. The mutated residues were selected based on sequence alignment and structural comparisons of several outer-membrane receptors. The quantitative determination of the dissociation constants in the presence of a respiratory inhibitor showed normal binding of the ferricanguibactin complex for all the mutants examined, which is concomitant with the selected residues being located below the binding site. The binding affinity of FatA to ferricanguibactin is about 100-fold lower than those described for the E. coli FepA and ferric enterobactin (Chakraborty et al., 2003; Annamalai et al., 2004) as well as for FhuA and ferrichrome (Annamalai et al., 2004) and similar to the low transport rates for enterobactin and corynebactin described by Jin et al. (2006) in the Gram-positive L. monocytogenes. Whether the low transport rate observed in the FatA receptor of $V$. anguillarum is an intrinsic characteristic of this receptor or a general characteristic of this species' ferricsiderophore receptors remains to be determined. 
Studies performed on the E. coli outer-membrane receptors FepA, FecA, FhuA and BtuB indicated that some regions of these proteins are conserved, as assessed not only by sequence alignments but also by structural analysis (Chimento et al., 2005). In fact, the important feature of these particular regions is that they are structurally conserved in location, orientation and bonding pattern in all these proteins. Therefore using molecular modelling, we identified amino acid residues that could form the lock region' quadrupole in FatA. Our modelling suggests that this region contains the charged residues R95, K130, E505 and E550, which form two separate points of contact between the plug or globular domain of the receptor and the $\beta$ barrel, which keep the globular domain in its place within the $\beta$ barrel. We were able to confirm this prediction by sitedirected mutagenesis and uptake kinetics experiments. The transport kinetics of these mutants indicated that arginine 95 and lysine 130, located in the quadrupole, are essential for ferric-anguibactin transport because mutations of these residues to both alanine and glutamine resulted in a complete loss of transport. Moreover, the binding properties of these mutants were similar to that of the wild-type, indicating that the variant receptors are properly folded in the outer membrane. In the case of FepA, the conserved R75 when changed to glutamine showed a strong adverse effect on transport, suggesting that it must be essential for the 'lock region'. The determinations performed previously on the $E$. coli ferrichrome receptor FhuA, in contrast, indicated that the substitution of R133 to alanine did not have any effect on transport (Endriss et al., 2003).

Conversely, when the conserved glutamic acid E505 on FatA was mutated to alanine or glutamine, no defect in transport was observed. Mutations in the E550 residue demonstrated its essential role in ferric-anguibactin transport. The substitutions of the glutamic acids by glutamines would be permissive for the formation of hydrogen bonds. Therefore, these hydrogen bonds might replace the salt bridges that are present in the wild-type receptor. However, substitution of a simple aliphatic residue, as is the case for alanine, would not result in the formation of hydrogen bonds. This prediction is corroborated by the results of the site-directed mutants. In another outer-membrane receptor, FepA, substitutions of residue E567 for glutamine results in a greater defect in transport when compared with substitutions in E511 (Chakraborty et al., 2003). Similar results were obtained in the case of the FatA E505A and E505Q residues. In the ferrichrome receptor FhuA, substitutions of the conserved glutamates E522 and E571 by alanines did not show any defect in transport (Endriss et al., 2003). Similar results were obtained when the E541 residue of FecA was substituted by alanine. Substitution of glutamate E587 by alanine in this receptor resulted in reduced transport rates as compared with the wild-type FecA (Sauter \& Braun, 2004).

Comparison of our results with those described for FepA, FhuA and FecA led us to hypothesize that the outer-membrane receptor quadrupoles might have some similarities and particularities in their involvement in the transport process. Although some results suggest that the transport mechanism would be comparable for both $V$. anguillarum and E. coli cells, only some of the residues of the quadrupole appeared to be essential for the transport of a specific ferric-siderophore complex. Taking into account our results together with those described for FepA, FhuA and FecA, it is therefore possible to speculate that the essentiality of some of the quadrupole amino acid residues belonging to the 'lock region' might depend on the type of siderophore, because the size and chemical properties of each ferric-siderophore complex are fairly different.

Two different theories explain the passage of the ferricsiderophore complex through the outer-membrane receptors. One of them postulates that the plug domain remains within the $\beta$-barrel and that during ferric-siderophore transport, allosteric transitions of the plug domain and the $\beta$ barrel would result in the opening of a channel through which the ligand permeates into the periplasm (Ferguson et al., 2002). Conversely, another theory proposes that the plug domain is removed from the barrel during the passage of the ferric-siderophore. Recently, the three-dimensional structure of the E. coli TonB protein in complex with the cobalamine receptor BtuB (Shultis et al., 2006) and the ferrichrome receptor FhuA (Pawelek et al., 2006) were solved. In both articles the authors suggest that a conformational change or localized unfolding of the plug domain must occur to open a permeation path that would allow siderophore translocation into the periplasm. Only two studies performed in FhuA (Eisenhauer et al., 2005; Endriss et al., 2003) suggest that a partial unfolding of the plug domain occurs during transport through this outermembrane receptor. However, whether the plug is partially or totally removed is still a matter of controversy. Although, the removal of the plug domain during TonB-dependent transport is unlikely to occur because this domain forms a large amount of intramolecular polar contacts with the $\beta$ barrel (Buchanan et al., 1999; Chimento et al., 2003; Ferguson et al., 1998, 2000, 2002, 2001; Locher et al., 1998; Yue et al., 2003), in accordance with Faraldo-Gömez et al. (2003) and Chimento et al. (2005), the extensive solvation of the barrel-plug interface might offer a solution to this problem.

In this study we postulate that the glycines present in the $\beta 5$ $\beta 6$ loop of the outer-membrane receptors are important for the conformational change or partial unfolding necessary to open a channel that allows transport through the barrel lumen. In contrast to other amino acids, glycines have a larger degree of freedom and a much larger range of allowed values for the phi/psi values in a Ramachandran plot. Therefore, rotations around glycines are favoured when a conformational change takes place within a protein or domains, since they serve as hinges between different domains. In the structure of FepA there are two glycines located at positions G127 (i) and G134 (i+7) that are part of the loop connecting the $\beta$ strands $\beta 5$ and $\beta 6$, located in the 
middle two strands of the central mixed $\beta$-sheet of the plug domain (Chakraborty et al., 2003, and Fig. 5a). The results obtained with FepA indicated that single mutations of these two conserved glycines to alanine affected transport but not binding. Moreover, only the double mutant of G127A (i)/G134A ( $i+7)$ showed a 12 -fold increase in the $K_{\mathrm{m}}$ value without affecting binding (Chakraborty et al., 2003). In the case of the $V$. anguillarum FatA receptor, single mutations at positions G131 (i), G138 (i+7) and G143 did not affect transport; however, it was affected by a triple mutation of these conserved residues. Furthermore, double mutations either did not affect transport, such as the G131A G138A and G138A G143A mutations, or abolished it, as was the case for the G131A G143A mutation. Altogether these results are more consistent with a model in which the conserved glycines located in the $\beta 5$ - $\beta 6$ loop could allow the rotation of these strands to form a transient channel (Fig. 5b). This model could be easily integrated with that in which a conformational change or partial unfolding of the plug might occur during ferric-siderophore transport. Since mutations of these three conserved glycines to alanine will result in small local changes in the FatA structure, it is unlikely that they will impede the complete removal of the whole plug domain. However, at this point we cannot rule out the possibility that a partial removal of the plug might occur during the transport of the ferric-anguibactin complex. Experiments to test our hypotheses are currently being performed.

\section{ACKNOWLEDGEMENTS}

We are grateful to Drs Dick van der Helm and Lothar Esser for their contribution in designing the FatA model and to Dr van der Helm for stimulating discussions. We are also grateful to Dr M. Jalal for advice concerning anguibactin purification. This work was supported by grants AI19018 and GM64600 from the National Institutes of Health to J.H. C.

\section{REFERENCES}

Actis, L. A., Fish, W., Crosa, J. H., Kellerman, K., Ellenberger, S. R., Hauser, F. M. \& Sanders-Loehr, J. (1986). Characterization of anguibactin, a novel siderophore from Vibrio anguillarum 775(pJM1). J Bacteriol 167, 57-65.

Actis, L. A., Tolmasky, M. E., Farrell, D. H. \& Crosa, J. H. (1988), Genetic and molecular characterization of essential components of the Vibrio anguillarum plasmid-mediated iron-transport system. J Biol Chem 263, 2853-2860.

Actis, L. A., Tolamsky, M. E. \& Crosa, J. H. (1999). Vibriosis. Wallingford, UK: CAB International Publishing.

Alice, A. F., Lopez, C. S. \& Crosa, J. H. (2005). Plasmid- and chromosome-encoded redundant and specific functions are involved in biosynthesis of the siderophore anguibactin in Vibrio anguillarum 775: a case of chance and necessity? J Bacteriol 187, 2209-2214.

Annamalai, R., Jin, B., Cao, Z., Newton, S. M. \& Klebba, P. E. (2004). Recognition of ferric catecholates by FepA. J Bacteriol 186, 3578-3589.
Bassford, P. J., Jr, Bradbeer, C., Kadner, R. J. \& Schnaitman, C. A. (1976). Transport of vitamin B12 in tonB mutants of Escherichia coli. J Bacteriol 128, 242-247.

Bradbeer, C. (1993). The proton motive force drives the outer membrane transport of cobalamin in Escherichia coli. J Bacteriol 175, 3146-3150.

Brunger, A. T., Adams, P. D., Clore, G. M., DeLano, W. L., Gros, P., Grosse-Kunstleve, R. W., Jiang, J. S., Kuszewski, J., Nilges, M. \& other authors (1998). Crystallography and NMR system: a new software suite for macromolecular structure determination. Acta Crystallogr D Biol Crystallogr 54, 905-921.

Buchanan, S. K., Smith, B. S., Venkatramani, L., Xia, D., Esser, L., Palnitkar, M., Chakraborty, R., van der Helm, D. \& Deisenhofer, J. (1999). Crystal structure of the outer membrane active transporter FepA from Escherichia coli. Nat Struct Biol 6, 56-63.

Bullen, J. J. \& Griffiths, E. (1999). Iron binding proteins and host defense. In Iron and Infection, pp. 327-368. Edited by J. J. Bullen \& E. Griffiths. Wiley.

Cao, Z., Qi, Z., Sprencel, C., Newton, S. M. \& Klebba, P. E. (2000). Aromatic components of two ferric enterobactin binding sites in Escherichia coli FepA. Mol Microbiol 37, 1306-1317.

Chakraborty, R., Lemke, E. A., Cao, Z., Klebba, P. E. \& van der Helm, D. (2003). Identification and mutational studies of conserved amino acids in the outer membrane receptor protein, FepA, which affect transport but not binding of ferric-enterobactin in Escherichia coli. Biometals 16, 507-518.

Chimento, D. P., Mohanty, A. K., Kadner, R. J. \& Wiener, M. C. (2003). Crystallization and initial X-ray diffraction of BtuB, the integral membrane cobalamin transporter of Escherichia coli. Acta Crystallogr D Biol Crystallogr 59, 509-511.

Chimento, D. P., Kadner, R. J. \& Wiener, M. C. (2005). Comparative structural analysis of TonB-dependent outer membrane transporters: implications for the transport cycle. Proteins 59, 240-251.

Cobessi, D., Celia, H. \& Pattus, F. (2005a). Crystal structure at high resolution of ferric-pyochelin and its membrane receptor FptA from Pseudomonas aeruginosa. J Mol Biol 352, 893-904.

Cobessi, D., Celia, H., Folschweiller, N., Schalk, I. J., Abdallah, M. A. \& Pattus, F. (2005b). The crystal structure of the pyoverdine outer membrane receptor FpvA from Pseudomonas aeruginosa at 3.6 angstroms resolution. J Mol Biol 347, 121-134.

Crosa, J. H. (1980). A plasmid associated with virulence in the marine fish pathogen Vibrio anguillarum specifies an iron-sequestering system. Nature 284, 566-568.

Crosa, J. H. \& Hodges, L. L. (1981). Outer membrane proteins induced under conditions of iron limitation in the marine fish pathogen Vibrio anguillarum 775. Infect Immun 31, 223-227.

Crosa, J. H. \& Walsh, C. T. (2002). Genetics and assembly line enzymology of siderophore biosynthesis in bacteria. Microbiol Mol Biol Rev 66, 223-249.

Datsenko, K. A. \& Wanner, B. L. (2000). One-step inactivation of chromosomal genes in Escherichia coli K-12 using PCR products. Proc Natl Acad Sci U S A 97, 6640-6645.

Eisenhauer, H. A., Shames, S., Pawelek, P. D. \& Coulton, J. W. (2005). Siderophore transport through Escherichia coli outer membrane receptor FhuA with disulfide-tethered cork and barrel domains. J Biol Chem 280, 30574-30580.

Endriss, F., Braun, M., Killmann, H. \& Braun, V. (2003). Mutant analysis of the Escherichia coli FhuA protein reveals sites of FhuA activity. J Bacteriol 185, 4683-4692.

Faraldo-Gomez, J. D., Smith, G. R. \& Sansom, M. S. (2003). Molecular dynamics simulations of the bacterial outer membrane 
protein FhuA: a comparative study of the ferrichrome-free and bound states. Biophys J 85, 1406-1420.

Ferguson, A. D. \& Deisenhofer, J. (2002). TonB-dependent receptors - structural perspectives. Biochim Biophys Acta 1565, 318-332.

Ferguson, A. D., Hofmann, E., Coulton, J. W., Diederichs, K. \& Welte, W. (1998). Siderophore-mediated iron transport: crystal structure of FhuA with bound lipopolysaccharide. Science 282, 2215-2220.

Ferguson, A. D., Braun, V., Fiedler, H. P., Coulton, J. W., Diederichs, K. \& Welte, W. (2000). Crystal structure of the antibiotic albomycin in complex with the outer membrane transporter FhuA. Protein Sci 9, 956-963.

Ferguson, A. D., Kodding, J., Walker, G., Bos, C., Coulton, J. W., Diederichs, K., Braun, V. \& Welte, W. (2001). Active transport of an antibiotic rifamycin derivative by the outer-membrane protein FhuA. Structure 9, 707-716.

Ferguson, A. D., Chakraborty, R., Smith, B. S., Esser, L., van der Helm, D. \& Deisenhofer, J. (2002). Structural basis of gating by the outer membrane transporter FecA. Science 295, 1715-1719.

Fischer, E., Gunter, K. \& Braun, V. (1989). Involvement of ExbB and TonB in transport across the outer membrane of Escherichia coli: phenotypic complementation of exb mutants by overexpressed ton $B$ and physical stabilization of TonB by ExbB. J Bacteriol 171, 5127-5134.

Frost, G. E. \& Rosenberg, H. (1975). Relationship between the tonB locus and iron transport in Escherichia coli. J Bacteriol 124, 704-712.

Guex, N. \& Peitsch, M. C. (1997). SWISS-MODEL and the SwissPdbViewer: an environment for comparative protein modeling. Electrophoresis 18, 2714-2723.

Jalal, M. A. F., Hossain, M. B., van der Helm, D., Sanders-Loehr, J., Actis, L. A. \& Crosa, J. H. (1989). Structure of anguibactin, a unique plasmid-related bacterial siderophore from the fish pathogen Vibrio anguillarum. J Am Chem Soc 111, 292-296.

Jin, B., Newton, S. M., Shao, Y., Jiang, X., Charbit, A. \& Klebba, P. E. (2006). Iron acquisition systems for ferric hydroxamates, haemin and haemoglobin in Listeria monocytogenes. Mol Microbiol 59, 1185-1198.

Kadner, R. J. (1990). Vitamin B12 transport in Escherichia coli: energy coupling between membranes. Mol Microbiol 4, 2027-2033.

Koster, W. L., Actis, L. A., Waldbeser, L. S., Tolmasky, M. E. \& Crosa, J. H. (1991). Molecular characterization of the iron transport system mediated by the pJM1 plasmid in Vibrio anguillarum 775. J Biol Chem 266, 23829-23833.

Locher, K. P., Rees, B., Koebnik, R., Mitschler, A., Moulinier, L., Rosenbusch, J. P. \& Moras, D. (1998). Transmembrane signaling across the ligand-gated FhuA receptor: crystal structures of free and ferrichrome-bound states reveal allosteric changes. Cell 95, 771-778.

Milton, D. L., O'Toole, R., Horstedt, P. \& Wolf-Watz, H. (1996). Flagellin A is essential for the virulence of Vibrio anguillarum. J Bacteriol 178, 1310-1319.

Mislin, G. L., Hoegy, F., Cobessi, D., Poole, K., Rognan, D. \& Schalk, I. J. (2006). Binding properties of pyochelin and structurally related molecules to FptA of Pseudomonas aeruginosa. J Mol Biol 357, $1437-1448$

Morales, V. M., Backman, A. \& Bagdasarian, M. (1991). A series of wide-host-range low-copy-number vectors that allow direct screening for recombinants. Gene 97, 39-47.

Pawelek, P. D., Croteau, N., Ng-Thow-Hing, C., Khursigara, C. M., Moiseeva, N., Allaire, M. \& Coulton, J. W. (2006). Structure of TonB in complex with FhuA, E. coli outer membrane receptor. Science 312, 1399-1402.

Postle, K. \& Kadner, R. J. (2003). Touch and go: tying TonB to transport. Mol Microbiol 49, 869-882.
Sambrook, B., Fritsh, E. F. \& Maniatis, T. (1989). Molecular Cloning: a Laboratory Manual. Cold Spring Harbor, NY: Cold Spring Harbor Laboratory.

Sauter, A. \& Braun, V. (2004). Defined inactive FecA derivatives mutated in functional domains of the outer membrane transport and signaling protein of Escherichia coli K-12. J Bacteriol 186, 5303-5310.

Schwyn, B. \& Neilands, J. B. (1987). Universal chemical assay for the detection and determination of siderophores. Anal Biochem 160, $47-56$.

Senanayake, S. D. \& Brian, D. A. (1995). Precise large deletions by the PCR-based overlap extension method. Mol Biotechnol 4, 13-15.

Shen, J. S., Geoffroy, V., Neshat, S., Jia, Z., Meldrum, A., Meyer, J. M. \& Poole, K. (2005). FpvA-mediated ferric pyoverdine uptake in Pseudomonas aeruginosa: identification of aromatic residues in FpvA implicated in ferric pyoverdine binding and transport. $J$ Bacteriol 187, 8511-8515.

Shultis, D. D., Purdy, M. D., Banchs, C. N. \& Wiener, M. C. (2006). Outer membrane active transport: structure of the BtuB:TonB complex. Science 312, 1396-1399.

Simon, R., Priefer, U. \& Puhler, A. (1983). A broad host range mobilization system for in vivo genetic engineering transposon mutagenesis in gram negative bacteria. Bio/Technology 1, 787-796.

Stork, M., Di Lorenzo, M., Mourino, S., Osorio, C. R., Lemos, M. L. \& Crosa, J. H. (2004). Two tonB systems function in iron transport in Vibrio anguillarum, but only one is essential for virulence. Infect Immun 72, 7326-7329.

Thompson, J. D., Higgins, D. G. \& Gibson, T. J. (1994). CLUSTAL W: improving the sensitivity of progressive multiple sequence alignment through sequence weighting, position-specific gap penalties and weight matrix choice. Nucleic Acids Res 22, 4673-4680.

Tolmasky, M. E. \& Crosa, J. H. (1984). Molecular cloning and expression of genetic determinants for the iron uptake system mediated by the Vibrio anguillarum plasmid pJM1. J Bacteriol 160, 860-866.

Tolmasky, M. E., Actis, L. A. \& Crosa, J. H. (1988). Genetic analysis of the iron uptake region of the Vibrio anguillarum plasmid pJM1: molecular cloning of genetic determinants encoding a novel trans activator of siderophore biosynthesis. J Bacteriol 170, 1913-1919.

van der Helm, D. (2004). Structure of outer membrane receptor proteins. In Iron Transport in Bacteria, pp. 51-65. Edited by A. R. Mey, S. M. Payne \& J. H. Crosa. Washington, DC: American Society for Microbiology.

van der Helm, D., Chakraborty, R., Ferguson, A. D., Smith, B. S., Esser, L. \& Deisenhofer, J. (2002). Bipartite gating in the outer membrane protein FecA. Biochem Soc Trans 30, 708-710.

Wertheimer, A. M., Verweij, W., Chen, Q., Crosa, L. M., Nagasawa, M., Tolmasky, M. E., Actis, L. A. \& Crosa, J. H. (1999). Characterization of the angR gene of Vibrio anguillarum: essential role in virulence. Infect Immun 67, 6496-6509.

Wiener, M. C. (2005). TonB-dependent outer membrane transport: going for Baroque? Curr Opin Struct Biol 15, 394-400.

Yu, D., Ellis, H. M., Lee, E. C., Jenkins, N. A., Copeland, N. G. \& Court, D. L. (2000). An efficient recombination system for chromosome engineering in Escherichia coli. Proc Natl Acad Sci U S A 97, 5978-5983.

Yue, W. W., Grizot, S. \& Buchanan, S. K. (2003). Structural evidence for iron-free citrate and ferric citrate binding to the TonB-dependent outer membrane transporter FecA. J Mol Biol 332, 353-368.

Edited by: J. Tommassen 